\title{
Reproductive Human Cells, Tissues, and Cellular and Tissue-Based Products from Anonymous Donor
}

National Cancer Institute

\section{Source}

National Cancer Institute. Reproductive Human Cells, Tissues, and Cellular and Tissue-

Based Products from Anonymous Donor. NCI Thesaurus. Code C133314.

Reproductive Human Cells, T issues and T issue based Products (HCT/Ps) donated by a donor to a recipient who doesn't know and isn't known by the recipient before donation. 